CARPATHIAN J. MATH.

Volume 38 (2021), No. 2,

Pages $391-404$
Online version at https : //semnul.com/carpathian/

Print Edition: ISSN 1584 - 2851; Online Edition: ISSN 1843 - 4401

DOI: https://doi.org/10.37193/CJM.2022.02.10

\title{
About convex structures on metric spaces
}

\author{
MitrofAn M. CHOBAN
}

\begin{abstract}
In the present paper we study the relationships between different concepts of convex structures in metric spaces that that are related to the works of K. Menger [Menger, K. Untersuchungen über allgemeine Metrik. Math. Ann. 100 (1928), 75-163], H. Busemann [Busemann, H. The geometry of geodesics, Academic Press, 1955], I. N. Herstein; J. Milnor [Herstein, I. N.; Milnor, J. An axiomatic approach to measurable utility. Econometrica 21 (1953), 291-297], E. Michael [Michael, E. Convex structures and continuous selections. Canad. J. Math. 11 (1959), 556-575], A. Nijenhuis [Nijenhuis, A. A note on hyperconvexity in Riemannian manifolds. Canad. J. Math. 11 (1959), 576-582.], W. Takahashi; T. Shimizu [Shimizu, T.; Takahashi, W. Fixed points of multivalued mappings in certain metric spaces. Topol. Methods Nonlinear Anal. 8 (1996), no. 1, 197-203 and Takahashi, W. A convexity in metric space and nonexpansive mappings I. Kodai Math. Sem. Rep. 22 (1970), 142-149], M. Tasković [Tasković, M. General convex topological spaces and fixed points. Math. Moravica 1 (1997), 127-134], Yu. A. Aminov [Aminov, Yu. A. Two-Dimensional Surfaces in 3-Dimensional and 4-Dimensional Euclidean Spaces. Results and Unsolved Problems. Ukr. Math. J. 71 (2019), no. 1, 1-38.], H. V. Machado [Machado, H. V. A characterization of convex subsets of normed spaces.Kodai Math. Sem. Rep. 25 (1973), 307-320], and many other papers.

Some well known examples of concrete convex structures are reexamined and the possibilities of different embeddings of metric spaces with convex structures are also studied. Corollary 6.1 states that the BolyaiLobachevskii plane and the Bolyai-Lobachevskii half-plane are not isometrically embedding in some strictly convex normed space. A characteristic of the invariant metric generated by a norm is presented (Proposition 4.6).
\end{abstract}

\section{INTRODUCTION}

The concept of distance and, in particular, of the metric is important in many branches of mathematics and applications. The main goal of this article is to consider examples of convex metrics that differ significantly from the convex structures generated by isometric embedding of a given space into some normed space. Some kinds of the convexities may be considered on spaces with general distances.

In the present article we consider only symmetrical distances in the following sense.

Definition 1.1. A distance space is a pair $(X, \rho)$ consisting of a set $X$ and a non-negative real-valued function $\rho$ on the set $X \times X$ satisfying the following conditions:

(D) $\rho(x, y)=0$ if and only if $x=y$;

(S) $\rho(x, y)=\rho(y, x)$, for all $x, y \in X$.

Let $(X, \rho)$ be a distance space. For any $x \in X$ and each $r>0$ we put

$$
\begin{aligned}
& B(x, r, \rho)=\{y \in X: \rho(x, y)<r\}, \\
& C(x, r, \rho)=\{y \in X: \rho(x, y) \leq r\} .
\end{aligned}
$$

The set $B(x, r, \rho)$ is the $\rho$-open ball with center $x$ and radius $r$ or, simply, the $r$-ball about $x$ and the set $C(x, r, \rho)$ is the $\rho$-closed ball with center $x$ and radius $r$ or, simply, the closed $r$-ball about $x$.

Received: 13.02.2021. In revised form: 17.05.2021. Accepted: 24.05.2021

2010 Mathematics Subject Classification. 52A01, 52A07, 51K05.

Key words and phrases. Convex structure, general convex topological spaces, metric space, embedding, immersion. 
The set $[a, b]_{\rho}=\{x \in X: \rho(a, b)=\rho(a, x)+\rho(x, b)\}$ is the $\rho$-interval with the ends $a, b \in X$. Obviously, from condition $(S)$ it follows that $[a, b]_{\rho}=[b, a]_{\rho}$.

We say that a set $U \subseteq X$ is $\rho$-open if for any $x \in U$ we have $B(x, \epsilon, \rho) \subseteq U$ for some $\epsilon>0$. The family $\mathcal{T}(\rho)$ of all $\rho$-open sets forms the topology of the distance space $(X, \rho)$, i.e., the topology induced by the distance $\rho$.

The open ball $B(x, r, \rho)$ is not obligatory an open subset of the topological space $(X, T(\rho))$ and the closed ball $C(x, r, \rho)$ is not obligatory a closed subset of the topological space $(X, T(\rho))$ (see [13]). We put $B^{0}(x, r, \rho)=\cup\{U \in T(\rho): U \subseteq B(x, r, \rho)\}$.

A distance space $(X, \rho)$ is called a strong distance space if, for any $x \in X$ and any $r>0$, we have $x \in B^{0}(x, r, \rho) \subseteq B(x, r, \rho)$. A distance space $(X, \rho)$ is a strong distance space if and only if the space $(X, T(\rho))$ is first-countable. Various concepts in the theory of distance spaces were discussed in $[2,5,29,30,31,32]$.

Example 1.1. Let $Z=\{(x, y): x, y \in \mathbb{R}, 0<x \leq 1,0<y \leq 1\}$ and $Y=\{(0,0)\} \cup Z$. For $(x, y),(u, v) \in Z$ we put $d((x, y),(u, v))=\sqrt{(x-u)^{2}+(y-v)^{2}}$. For any $(x, y) \in Y$ we put $d((x, y),(0,0))=d((0,0),(x, y))=x$ for $y=0$ and $d((x, y),(0,0))=d((0,0),(x, y))=2$ for $y>0$. In this case, $B((x, y), r, d)$ is an open subset of $(Y, T(d))$, for any point $(x, y) \neq(0,0)$ and $r>0$, and $B^{0}((0,0), r, d)=\emptyset$, for any $r \leq 2$. Let $Y_{n}=Y$ for any $n \in \mathbb{N}$. We put $X$ $=\left\{\left(y_{n}: n \in \mathbb{N}\right) \in Y^{\mathbb{N}}:\right.$ the set $\left\{n \in \mathbb{N}: y_{n} \neq(0,0)\right\}$ is finite $\}$ and $\rho\left(\left(\left(y_{n}: n \in \mathbb{N}\right),\left(z_{n}:\right.\right.\right.$ $n \in \mathbb{N}))=\Sigma\left\{2^{-n} d\left(y_{n}, z_{n}\right): n \in \mathbb{N}\right\}$, for any two points $\left(y_{n}: n \in \mathbb{N}\right),\left(z_{n}: n \in \mathbb{N}\right) \in X$. In this case, for any point $x=\left(x_{n}: n \in \mathbb{N}\right) \in X$ we put $\operatorname{ind}(x)=\min \left\{n \in \mathbb{N}: x_{n}=(0,0)\right\}$. Then, for $r \leq 2^{-i n d(x)}$, we have $B(x, r, \rho)=\emptyset$. In particular, the space $(X, \rho)$ is Lindelöf, completely regular and without first axiom of countability at each point. Moreover, the space $X$ is $\sigma$-compact and a continuous image of a separable complete metrizable space.

Definition 1.2. A distance space $(X, \rho)$ is said to be:

1) a metric space [20] if it satisfies the following condition:

$\rho(x, z) \leq \rho(x, y)+\rho(y, z)$, for all $x, y, z \in X$;

2) a b-metric space [3] if there exists a number $\lambda>0$ such that $d(x, z) \leq \lambda(d(x, y)+$ $d(y, z))$, for all $x, y, z \in X$.

3) an $F$-distance space $[20,11]$ if, for any $\varepsilon>0$, there exists a $\delta>0$ such that $\rho(x, z)<\varepsilon$ provided $\rho(x, y)<\delta$ and $\rho(y, z)<\delta$ for some $y \in Y$;

4) a $N$-distance space [33] if, for any point $x \in X$ and each $\varepsilon>0$ there exists a $\delta>0$ such that $\rho(x, z)<\varepsilon$ provided $\rho(x, y)<\delta$ and $\rho(y, z)<\delta$ for some $y \in Y$;

5) a $\Pi$-distance space [33] if, for any point $x \in X$ and any two sequences $\left\{x_{n} \in X: n \in\right.$ $\mathbb{N}\}$ and $\left\{y_{n} \in X: n \in \mathbb{N}\right\}$ we have $\lim _{n \rightarrow \infty} \rho\left(x, y_{n}\right)=0$ provided $\lim _{n \rightarrow \infty} \rho\left(x, x_{n}\right)=0$ and $\lim _{n \rightarrow \infty} \rho\left(x_{n}, y_{n}\right)=0$ for some $y \in Y$;

6) a $W$-metric space [41] if, for each point $X \in X$ and each positive number $k$, there is a positive number $r$ such that if $y \in X$ is a point for which $\rho(x, y) \geq k$, then $\rho(x, z)+\rho(y, z) \geq$ $r$, for each $z \in X$.

Any metric space is a $b$-metric space and any $b$-metric space is an $F$-distance space (see $[3,5])$. From the E. W. Chittenden's theorem $[11,20]$ any $F$-distance space is metrizable. Moreover, for any $F$-distance $\rho$ on a set $X$ there exists a metric $d$ on $X$ such that, for any $\varepsilon>0$, there exists a $\delta>0$ for which $\rho(x, y)<\varepsilon$ provided $d(x, y)<\delta$ and vice-versa $d(x, y)<\varepsilon$, provided $\rho(x, y)<\delta$.

V. V. Niemytzki [33] proved that any $N$-distance is a $\Pi$-distance, any $\Pi$-distance is a $N$ distance and any $N$-distace space is metrizable (see [29, 30, 31, 32]). In [41] W. A. Wilson proved that any $W$-distance is a $N$-distance, any $N$-distance is a $W$-distance and any $W$ distace space is metrizable.

The notion of metric convexity due to K. Menger [27].

The term convexity is very diversified and it is used in various branches of mathematics. 
Let $\mathbb{R}$ be the space of reals and $\mathbb{R}^{n}$ be the $n$-dimensional vector space with the Euclidean metric $d_{2}^{n}\left(\left(x_{1}, x_{2}, \ldots, x_{n}\right),\left(y_{1}, y_{2}, \ldots, y_{n}\right)\right)=\sqrt{\Sigma\left\{\left(y_{i}-x_{i}\right)^{2}: i \leq n\right\}}$. The set $\mathbb{I}=\{t: 0 \leq t \leq 1\}$ is the unit segment of the space $\mathbb{R}^{1}$.

On the space $\mathbb{R}^{n}$, for any number $p>0$, we consider the following distances

$$
\left.d_{p}^{n}\left(\left(x_{1}, x_{2}, \ldots, x_{n}\right),\left(y_{1}, y_{2}, \ldots, y_{n}\right)\right)=\left(\Sigma\left\{\left|y_{i}-x_{i}\right|^{p}: i \leq n\right\}\right\}\right)^{1 / p}
$$

and

$$
\left.d_{\infty}^{n}\left(\left(x_{1}, x_{2}, \ldots, x_{n}\right),\left(y_{1}, y_{2}, \ldots, y_{n}\right)\right)=\sup \left\{\left|y_{i}-x_{i}\right|: i \leq n\right\}\right\} .
$$

For $p<1$, the distance $d_{p}^{n}$ is a $b$-metric with $\lambda=2^{1 / p}[4]$, while for $p \geq 1$, the distance $d_{p}^{n}$ is a metric.

Definition 1.3. A convex structure on a distance space $(E, d)$ assigns a mapping $\mu: E \times$ $E \times \mathbb{I} \longrightarrow E$ with the properties:

1) $\mu(x, y, t)=\mu(y, x, 1-t)$, for all $x, y \in E$ and $t \in \mathbb{I}$;

2) $d(x, \mu(x, y, t))=(1-t) \cdot d(x, y)$ and $d(\mu(x, y, t), y)=t \cdot d(x, y)$, for all $x, y \in E$ and $t \in \mathbb{I}$.

3) for any $x, y \in E$ the mapping $\mu^{(x y)}: \mathbb{I} \longrightarrow E$, where $\mu^{(x y)}(t)=\mu(x, y, t)$ for any $t \in \mathbb{I}$, is continuous.

By definition, we have $\mu(x, y, 1)=x$ and $\mu(x, y, 0)=y$.

On any linear space $L$ we consider the linear convexity $\mu_{l}(x, y, t)=(1-t) \cdot x+t \cdot y$, for all $x, y \in E$ and $t \in \mathbb{I}$.

Definition 1.4. Let $\mu: E \times E \times \mathbb{I} \longrightarrow E$ be a convex structure on a distance space $(E, d)$.

1) For any two points $a, b \in E,[a, b]_{\mu}=\{\mu(a, b, t): t \in \mathbb{I}\}$ is the $\mu$-segment with the end points $a, b$.

2) A subset $A \subseteq E$ is called convex if $\mu(x, y, t) \in A$, for all $x, y \in E$ and $t \in \mathbb{I}$.

3) A subset $A \subseteq E$ is called $d$-convex or a metric subset if $[x, y]_{d} \subseteq A$, for all $x, y \in E$.

4) A convex distance space $(E, d, \mu)$ is a hereditary convex distance space if $\mu(x, y, t) \in[a, b]_{\mu}$, for for all $a, b \in E, x, y \in[a, b]_{\mu}$ and $t \in \mathbb{I}$.

We have $[a, b]_{\mu} \subseteq[a, b]_{d}$ for all points $a, b \in E$. Hence any metric convex set in the Menger's sense is convex. For $p \neq 2$ and $n \geq 2$ in the metric space $\left(\mathbb{R}^{n}, d_{p}^{n}\right)$ with the linear convexity there exists a convex set which is not metric convex. By definition, the empty set is $\rho$-convex. The concept of Menger's convexity is very large. By instance, on an discrete metric space $(E, d)$, where $d(x, x)=0$ and $d(x, y)=1$, for distinct $x, y \in E$, any subset $A \subset E$ is $d$-convex and on $(E, d)$ there are no convex structures. Various aspects of convex structures were studied and applied to certain problems by many authors, see $[6,8,10,12,17,26,28,38,39,40]$.

\section{UNIFORM CONVEX STRUCTURES}

In the case of the Fixed Point Theory, some convex structures with special metric constraints were introduced and used.

Definition 2.5. A convex structure $\mu: E \times E \times \mathbb{I} \longrightarrow E$ on a distance space $(E, d)$ is called:

1) uniformly continuous if, for every $\varepsilon>0$, there exist $\delta, \xi \in \mathbb{R}$ such that $\varepsilon \geq \xi \geq \delta>0$ and $d(\mu(x, y, t), \mu(s, z, t))<\xi$ provided $x, y, s, z \in E, t \in \mathbb{I}, d(x, s)<\xi$ and $d(y, z)<\delta$;

2) a convex structure with convex round balls if $d(z, \mu(x, y, t)) \leq \max \{d(z, x), d(z, y)\}$, for all $x, y, z \in E$ and $t \in \mathbb{I}$;

3) a strictly convex structure with convex round balls if $d(z, \mu(x, y, t))<\max \{d(z, x), d(z, y)\}$, for all $x, y, z \in E$ and $t \in \mathbb{I}, 0<t<1$, and for all $x, y \in E$ and $t \in \mathbb{I}$ there exists a unique point $z \in E$ such that $d(x, z)=t \cdot d(x, y)$ and $d(z, y)=(1-t) \cdot d(x, y)$; 
4) a T-convex structure if $d(z, \mu(x, y, t)) \leq t \cdot d(z, x)+(1-t) \cdot d(z, y)$, for all $x, y, z \in E$ and $t \in \mathbb{I}$.

5) a strongly $T$-convex structure if $d(z, \mu(x, y, t))<t \cdot d(z, x)+(1-t) \cdot d(z, y)$, for all distinct points $x, y, z \in E$ and $0<t<1$.

Convex structures, convex structures with convex round balls and strictly convex structures with convex round balls were studied in $[7,10,12,21,22,37]$, while $T$-convex structures were studied in $[35,36]$.

Definition 2.6. Let $0<\gamma \leq 1$. A convex structure $\mu: E \times E \times \mathbb{I} \longrightarrow E$ on a distance space $(E, d)$ is called:

- a hyperconvex structure if, for every positive number $\varepsilon>0$ and any points $a, b \in E$, there exists $\delta>0$ such that from $x, y \in E$ the inequalities $d(a, x)<\delta, d(b, y)<\delta$ imply that $d(\mu(x, y, t), \mu(u, v, t))<\varepsilon$ for any $t \in \mathbb{I}$.

- a $\gamma$-hyperconvex structure if, for every positive number $\varepsilon>0$ and any points $x, y, u, v \in$ $E$, the inequalities $d(x, u)<\varepsilon, d(y, v)<\gamma \cdot \varepsilon$ imply that $d(\mu(x, y, t), \mu(u, v, t))<\varepsilon$ between corresponding points of $t \in \mathbb{I}$.

Any $\gamma$-hyperconvex structure is a hyperconvex structure. The concept of $\gamma$-hyperconvex structure was introduced by A. Nijenhuis [34], who proved (Theorem II) that: if $(M, d)$ is a Riemann manifold with the convex metric, then:

1) if the curvature of $M$ is non-positive, then $M$ is 1-hyperconvex;

2) if the curvature is positive, then every point of the Riemannian manifold $M$ has a $2^{-1}$-hyperconvex neighborhood.

Remark 2.1. Any $T$-convex structure is a convex structure with convex round balls.

Remark 2.2. Any $\gamma$-hyperconvex structure is a uniformly continuous convex structure.

Remark 2.3. Any strictly convex structure with convex round balls is a hereditary convex structure with convex round balls. If $\mu$ is a hyperconvex structure on a metric space $(E, d)$, then $\mu: E \times E \times \mathbb{I} \longrightarrow E$ is a continuous mapping.

Proposition 2.1. Let $\mu: E \times E \times \mathbb{I} \longrightarrow E$ be a convex structure with convex round balls on a distance space $(E, d)$. Then $B(a, r, d)$ and $C(x, r, d)$ are convex subsets of the space $(E, d)$.

Proof. If $x, y \in B(a, r, d)$ and $t \in \mathbb{I}$, then $d(a, \mu(x, y, t)) \leq \max \{d(a, x), d(a, y)\}<r$ and $\mu(x, y, t) \in B(a, r, d)$. If $x, y \in C(a, r, d)$ and $t \in \mathbb{I}$, then $d(a, \mu(x, y, t)) \leq \max \{d(a, x), d(a, y)\}$ $\leq r$ and $\mu(x, y, t) \in C(a, r, d)$.

If $E$ is a linear space, then $[a, b]=\{t \cdot a+(1-t) \cdot b: t \in \mathbb{I}\}$ is the linear segment with the end points $a, b$. If $(E, n)$ is a normed space and $d(x, y)=n(x-y)$, then $[a, b]=[a, b]_{\mu_{l}} \subseteq[a, b]_{d}$.

Let $(X, d, \mu)$ be a metric space with a convex structure. A subset $L \subseteq X$ is $\mu$-collinear if, for any three points $x, y, z \in L$, there exist two points $a, b \in X$ such that $x, y, z \in[a, b]_{\mu}$. A maximal $\mu$-collinear subset of $X$ is called a $\mu$-line.

Proposition 2.2. Let $(X, d, \mu)$ be a metric space with a hereditary convex structure. Then for any two distinct points $a, b \in X$ there exists some $\mu$-line $(a b)_{\mu}$ such that $a, b \in(a b)_{\mu}$.

Proof. Fix two distinct points $a, b \in X$. There exists a maximal family of $\mu$-segments $\mathcal{L}(a, b)=\left\{[x, y]_{\mu}: x, y \in X,[a, b]_{\mu} \subseteq[x, y]_{\mu}\right\}$ linearly ordered by inclusion. We put $(a b)_{\mu}$ $=\cup \mathcal{L}(a, b)$. Assume that $x, y \in(a b)_{\mu}$. Then there exist four points $x_{1}, x_{2}, y_{1}, y_{2} \in X$ such that:

$$
\begin{aligned}
& \text { - } x \in\left[x_{1}, x_{2}\right]_{\mu} \in \mathcal{L}(a, b) ; \\
& \text { - } y \in\left[y_{1}, y_{2}\right]_{\mu} \in \mathcal{L}(a, b) .
\end{aligned}
$$


Then one of the $\mu$-segments $\left[x_{1}, x_{2}\right]_{\mu},\left[y_{1}, y_{2}\right]_{\mu}$ is a subset of other. Thus the points $a, b, x, y$ are $\mu$-collinear. By induction we can proved that any finite set $L \subseteq[a, b]_{\mu}$ is $\mu$ collinear.

Fix a point $c \in X \backslash(a b)_{\mu}$. We have two possible cases.

Case 1. If $x, y \in X$ and $[a, b]_{\mu} \subseteq[x, y]_{\mu}$, then $c \notin[x, y]_{\mu}$.

In this case the points $a, b, c$ are not $\mu$-collinear.

Case 2. There exist two points $x, y \in X$ such that $[a, b]_{\mu} \subseteq[x, y]_{\mu}$ and $c \in[x, y]_{\mu}$.

Since $\mu$ is a hereditary convex structure, the intersection $(a b)_{\mu} \cap[x, y]_{\mu}$ is a $\mu$-segment. We can assume that $(a b)_{\mu} \cap[x, y]_{\mu}=[a, b]_{\mu}$. Since the family $\mathcal{L}(a, b)$ is maximal and $[x, y]_{\mu} \notin$ $\mathcal{L}(a, b)$, there exist two points $u, v \in X$ such that $[a, b]_{\mu} \subseteq[u, v]_{\mu} \in \mathcal{L}(a, b),\{u, v\} \backslash\{a, b\} \neq \emptyset$ and $c \notin[u, v]_{\mu}$. In this case the points $u, v, c$ are not $\mu$-collinear and for $x, y \in X$ and $[u, v]_{\mu} \subseteq[x, y]_{\mu}$ follows that $c \notin[x, y]_{\mu}$. Hence $(a b)_{\mu}$ is a $\mu$-line.

\section{ON BOLYAI-LOBACHEVSKII PLANE}

When the parallel postulate is removed from Euclidean geometry, the resulting geometry is the absolute geometry.

Denote by $L$ the absolute plane with the metric $d$. Assume that $L$ is the Bolyai-Lobachevskii plane or the Euclidean plane. Consider two distinct points $a, b \in L$. A point $x \in L$ is between the points $a$ and $b$ if and only if $x$ is a point of the straight line $(a b)$ passing through the distinct points $a, b$ and $d(a, b)=d(a, x)+d(x, b)$. For any number $t \in \mathbb{I}=[0,1]$ there exists a unique point $\mu_{L}(a, b, t)=x_{t}$ such that $d\left(a, x_{t}\right)=(1-t) \cdot d(a, b)$ and $d\left(x_{t}, b\right)=$ $t \cdot d(a, b)$. In particular, on a metric space $(L, d)$ there exists a unique convex structure $\mu_{L}: L \times L \times \mathbb{I} \longrightarrow L$ such that:

- $[a, b]_{d}=\{x \in L: d(a, x)+d(x, b)=d(a, b)$ for any two points $a, b \in L$;

- $[a, b]=\{x \in L: x$ is between the points $a, b\} \subseteq(a b)$ is the line segment with the end-points $a, b \in L$;

- $[a, b]=[a, b]_{d}=[a, b]_{\mu}$ for any two points $a, b \in L$.

Proposition 3.3. A convex structure $\mu_{L}: L \times L \times \mathbb{I} \longrightarrow L$ on the absolute plane $(L, d)$ is a uniformly continuous hereditary strictly convex structure with convex round balls.

Proof. There are two kinds of absolute geometry: Euclidean geometry and hyperbolic or Bolyai-Lobachevskii geometry. The case of Euclidean plane is well known.

Assume that $L$ is the Bolyai-Lobachevskii plane. Consider two distinct points $a, b \in L$. A point $x \in L$ is between the points $a$ and $b$ if and only if $x$ is a point of the straight line $(a b)$ passing through the distinct points $a, b$ and $d(a, b)=d(a, x)+d(x, b)$. For any number $t \in \mathbb{I}=[0,1]$ there exists a unique point $\mu_{L}(a, b, t)=x_{t}$ such that $d\left(a, x_{t}\right)=(1-t) \cdot d(a, b)$ and $d\left(x_{t}, b\right)=t \cdot d(a, b)$. In particular, on a metric space $(L, d)$ the convex structure $\mu_{L}$ : $L \times L \times \mathbb{I} \longrightarrow L$ is the unique convex structure such that:

- $[a, b]=[a, b]_{d}=[a, b]_{\mu}$ for any two points $a, b \in L$;

- if $a, b \in L$ and $u, v \in[a, b]_{d}$, then $[u, v]_{d}=[u, v]_{\mu} \subseteq[a, b]_{\mu}=[a, b]_{d}$.

Hence $\mu_{L}$ is a hereditary strictly convex structure on the space $(L, d)$.

Fix three distinct points $a, b, c \in L$ and a point $x \in L$ between the points $a$ and $b$. Assume that $c \notin[a, b]$.

If $c \in(a b)$, then $x \in[c, a]_{d} \cup[c, b]_{d}$. If $x \in[c, a]_{d}$, then $d(c, a)>d(c, x)$.

Suppose that $c \notin(a b)$. There is a perpendicular line $(c p)$ to a line $(a b)$ through the point $c$, where $p \in(a b)$. Fix a point $z \in(a, b)$. Then $c p z$ is a right triangle with the hypotenuse $[c z]_{d}$ and catheters $[c, z]_{d}$ and $[p z]_{d}$. Suppose that $d(c, a) \geq d(c, b)$.

Consider the hyperbolic function defined as $\cosh (z)=\frac{1}{2}\left(e^{z}+e^{-z}\right)$. By virtue of analogous of Pythagora's Theorem we have $\cosh (d(c, z))=\cosh (d(c, p)) \cdot \cosh (d(p z))$. Hence for 
$u \in(a, b)$ we have $d(c, z)<d(c, u)$ if and only if $d(p, z)<d(p, u)$. Since $z$ is a point strictly between $a, b$ and $d(c, a) \geq d(c, b)$, then $d(c, a)>d(c, z)$.

Therefore $\mu$ is a hereditary strictly convex structure with convex round balls.

Proposition 3.4. A convex structure $\mu: L \times L \times \mathbb{I} \longrightarrow L$ on the absolute plane $(L, d)$ is uniformly continuous.

Proof. Follows by A. Nijenhuis results [34].

Proposition 3.5. A convex structure $\mu_{L}: L \times L \times \mathbb{I} \longrightarrow L$ on the absolute plane $(L, d)$ is a strongly T-convex structure.

Proof. The assertion is true for the Euclidean plane $\left(\mathbb{R}^{2}, d_{2}^{2}\right)$.

Assume that $(L, d)$ is a hyperbolic space of non-positive curvature $k$. Fix a geodesic triangle $\triangle(a, b, c)$ in $L$ with vertices $a, b, c$. The sides $a b, a c, b c$ of the triangle $\triangle(a, b, c)$ are the geodesic lines of the length $d(a, b), d(a, c), d(b, c)$ respectively.

A comparison triangle in $\mathbb{R}^{2}$ for the geodesic triangle $\triangle(a, b, c)$ a triangle in the Euclidean plane with vertices $\bar{a}, \bar{b}, \bar{c}$ such that $d_{2}^{2}(\bar{a}, \bar{b})=d(a, b), d_{2}^{2}(\bar{a}, \bar{c})=d(a, c)$ and $d_{2}^{2}(\bar{b}, \bar{c})=$ $d(b, c)$. Such a triangle is unique up to isometry, and shall be denoted $\triangle(\bar{a}, \bar{b}, \bar{c})$. Fix a point $x$ on the side $b c$ such that $d(b, x)=(1-t) \cdot d(b, c)$ and $0<t<1$. Then $d(x, c)=t \cdot d(b, c)$. On the interval $\bar{b} \bar{c})$ there exists a unique points $\bar{x}$ such that $d_{2}^{2}(\bar{b}, \bar{x})=(1-t) \cdot d_{2}^{2}(\bar{b}, \bar{c})$. Since $L$ is a hyperbolic space of the non-positive curvature $k<0$, by virtue of $C A T(k)$ inequality, we have $d(a, x) \leq d_{2}^{2}(\bar{a}, \bar{x})[6,9,23]$. Since $\triangle(\bar{a}, \bar{b}, \bar{c})$ is an Euclidean triangle we have $d_{2}^{2}(\bar{a}, \bar{x}) \leq(1-t) d_{2}^{2}(\bar{a}, \bar{b})+t d_{2}^{2}(\bar{a}, \bar{c})$. Therefore $d(a, x) \leq d_{2}^{2}(\bar{a}, \bar{x}) \leq t \cdot d_{2}^{2}(\bar{a}, \bar{b})+(1-t) \cdot d_{2}^{2}(\bar{a}, \bar{c})$ $=t \cdot d(a, b)+(1-t) \cdot d(a, c)$.

\section{CONVEXITIES ON LINEAR SPACES}

Let $(B, n)$ be a normed liner space with the norm $n$ with the properties:

1) $n(x)=0$ if and only if $x=0$;

2) $n(t \cdot x)=|t| \cdot n(x)$, for all $x \in B$ and $t \in \mathbb{R}$;

3) $n(x+y) \leq n(x)+n(y)$, for all $x, y \in B$.

We put $d(x, y)=n(x-y)$ and $\mu_{B}(x, y, t)=t x+(1-t) y$, for all $x, y \in B$ and $t \in \mathbb{I}$. In this case $d$ is the metric generated by the norm $n(x)$ and $\mu$ is a convexity generated by the linear structure of the space $B$. The metric generated by a norm is invariant.

A metric $d$ on a linear space $B$ is invariant if it satisfies the following equivalent properties:

(i) $d(x+z, y+z)=d(x, y)$, for all $x, y, z \in B$;

(ii) $d(x, y)=d(x-y, 0)$, for all $x, y \in B$.

The convex metric space $\left(B, d, \mu_{B}\right)$ is a metric space with 1 -hyperconvexity and with a uniformly continuous hereditary convex structure with convex round balls. That convex structure is strictly convex structure with convex round balls provided $(B, n)$ is a uniformly convex Banach space. We have $d(t \cdot x, 0)=t \cdot d(x, 0)$ and $d(t \cdot x+(1-t) \cdot y, 0) \leq t \cdot d(x, 0)$ $+(1-t) \cdot d(y, 0)$, for all $x, y \in B$ and $t \in \mathbb{I}$.

Professor V. Berinde posed the following question: let $d$ be a metric on the linear space $B$ with the next two properties:

(1) $d$ is invariant, i.e. $d(x, y)=d(x-y, 0)$, for all $x, y \in B$;

(2) $d(t \cdot x+(1-t) \cdot y, 0) \leq t \cdot d(x, 0)+(1-t) \cdot d(y, 0)$, for all $x, y \in B$ and $t \in \mathbb{I}$.

Is it true that $n(x)=d(x, 0)$ is not a norm on the linear space?

The response is negative. We mention the following fact which, in particular, states that a metric on a linear space is generated by the norm provided it is in accordance with the linear convexity of the space. 
Proposition 4.6. Assume that $(B, d)$ is a linear space with an invariant metric $d$. The following properties are equivalent:

(1) $d(t \cdot x, 0)=t \cdot d(x, 0)$, for all $x \in B$ and $t \in \mathbb{I}$.

(2) $n(x)=d(x, 0)$ is a norm on the linear space $B$ and $d(x, y)=n(x-y)$, for all $x, y \in B$.

(3) the linear convex structure $\mu(x, y, t)=(1-t) \cdot x+t \cdot y$ is a convex structure on the metric space $(B, d)$.

(4) $d(t \cdot x, 0) \leq t \cdot d(x, 0)$, for all $x \in B$ and $t \in \mathbb{I}$.

(5) $d(t \cdot x+(1-t) \cdot y, 0) \leq t \cdot d(x, 0)+(1-t) \cdot d(y, 0)$, for all $x, y \in B$ and $t \in \mathbb{I}$.

Proof. Implications $(2) \rightarrow(3) \rightarrow(1) \rightarrow(4)$ and $2 \rightarrow(5) \rightarrow$ (4) are obvious.

Assume that $d(t \cdot x, 0)=t \cdot d(x, 0)$ for all $x \in B$ and $t \in \mathbb{I}$. We will prove a series of statements.

S1. $d(x+z, y+z)=d(-x,-y)=d(x, y)$ for all $x, y, z \in B$.

Really, $d(x+z, y+z)=d((x+z)-(y+z), 0)=d(x-y, 0)=d(x, y)$ and $d(x, y)=$ $d(x-y, y-y)=d(x-y, 0)=d(x-y-x, 0-x)=d(-y,-x)=d(-x,-y)$.

S2. $n(-x)=n(x)$ for each $x \in B$.

By definition, $n(-x)=d(-x, 0)$. By assertion S1 we have $n(-x)=d(-x, 0)=d(-(-x),-0)$ $=d(x, 0)=n(x)$.

S3. $n(t \cdot x)=n(|t| \cdot x)$ for all $x \in B$ and $t \in \mathbb{R}$.

Since $|t|=t$ for $t \geq 0$ and $|t|=-t$ for $t \leq 0$, assertion S3 follows from assertion S2.

S4. $n(x+y) \leq n(x)+n(y)$ for all $x, y \in B$.

From properties of metric it follows $n(x+y)=d(x+y, 0)=d(x,-y) \leq d(x, 0)+d(0,-y)$ $=n(x)+n(-y)=n(x)+n(y)$.

S5. $n(x)=0$ if and only if $x=0$.

If $x=0$, then $n(0)=d(0,0)=0$. Assume that $x \in B$ and $n(x)=0$. Then $0=n(x)=$ $d(x, 0)$ and $d(x, 0)=0$ if and only if $x=0$.

S6. $n(t \cdot x)=|t| \cdot n(x)$ for all $x \in B$ and $t \in \mathbb{R}$.

By virtue of assertion S3, we can assume that $t>0$. Consider three possible cases.

Case 1. $t=1$.

Since $1 \cdot x=x$, we have $n(1 \cdot x)=n(x)=|1| \cdot n(x)$.

Case 2. $0 \leq t<1$.

In this case $t \in \mathbb{I}$ and $n(t \cdot x)=d(t \cdot x, 0)=t \cdot d(x, 0)=|t| \cdot n(x)$.

Case 3. $t>1$.

In this case $0<t^{-1}<1$ and $n(x)=n\left(t^{-1} \cdot(t \cdot x)\right)=t^{-1} \cdot n(t \cdot x)$. Therefore $n(t \cdot x)=$ $|t| \cdot n(x)$.

S7. $n(x)$ is a norm on $B$ and $d(x, y)=n(x-y)$ for all $x, y \in B$.

Follows from assertions S4, S5, S6 and construction of the norm $n(x)$. Implications $(1) \rightarrow(2) \rightarrow(3)$ are proved.

Assume now that $n(x)=d(x-y, 0)$ is a norm on the linear space $B$. Since $n(t \cdot x)=$ $|t| \cdot n(x)$ for all $x \in B$ and $t \in \mathbb{R}$, we have $d(t \cdot x, 0=t \cdot d(x, 0)$ for all $x \in B$ and $t \in \mathbb{I}$.

Assume that $d(t \cdot x, 0) \leq t \cdot d(x, 0)$ for all $x \in B$ and $t \in \mathbb{I}$. Suppose that $\lambda \in \mathbb{I}$ and $d(\lambda \cdot a, 0)<\lambda \cdot d(a, 0)$ for some $a \in B$. Then $d(a, 0)=d(\lambda \cdot a+(1-\lambda) \cdot a, 0) \leq$ $d(\lambda \cdot a, 0)+d((1-\lambda) \cdot a, 0) \leq \lambda \cdot d(a, 0)+d((1-\lambda) \cdot a, 0) \leq \lambda \cdot d(a, 0)+(1-\lambda) \cdot d(a, 0)=$ $d(a, 0)$, a contradiction. So, implication $(4) \rightarrow(1)$ is proved.

\section{EMBEDDINGS OF CONVEX METRIC SPACES}

Various embeddings of metric spaces appear in the paper of M. Fréchet [19], where he first introduces the notion of metric space.

K. Kuratowski [25] proved the following embedding theorem. 
Theorem 5.1. (K. Kuratowski [25]) Let $(X, d)$ be a metric space, $\omega$ be a point in $X$ and $B=$ $C^{0}(X)$ denotes the Banach space of all bounded continuous real-valued functions on $X$ with the supremum norm $n(f)=\sup \{|f(x)|: x \in X\}$. Then the mapping $\Phi: X \longrightarrow B$, defined by $\Phi(a)(x)=d(x, a)-d(x, \omega)$ for all $a, x \in X$, is an isometric embedding and $\Phi(\omega)$ is the zero 0 of the linear space $C^{0}(X)$.

Proof. It is well known that set $B=C^{0}(X)$ of all bounded continuous real-valued functions on $X$ with the supremum norm $n(f)=\sup \{|f(x)|: x \in X\}$ is a Banach space. For any point $a \in X$ consider the continuous function $\varphi_{a}(x)=d(x, a)-d(x, \omega)$ for all $a, x \in X$. We have:

- $\Phi(a)=\varphi_{a}$ for any point $a \in X$;

- $\varphi_{\omega}(x)=0$ for any $x \in X$;

- $n\left(\varphi_{a}\right)=d(a, \omega)$ for any $a \in X$;

- $d(x, y)=n\left(\varphi_{x}-\varphi_{y}\right)$ for all $x, y \in X$.

Hence $\Phi: X \longrightarrow B$ is the desired isometric embedding. This embedding depends on the chosen point $\omega \in X$.

Let $(X, d, \mu)$ be a metric space with a convex structure. The following assertion shows that the Kuratowski's embedding $\Phi: X \longrightarrow C^{0}(X)$ as a rule does not accord the convexities $\mu$ on $X$ and $\mu_{l}$ on $C^{0}(X)$.

Proposition 5.7. Let $(X, d, \mu)$ be a metric space with a hereditary strictly convex structure with convex round balls and assume that $X$ is not a $\mu$-line. Let $\omega$ be a point in $X$ and denote by $B=$ $C^{0}(X)$ the Banach space of all bounded continuous real-valued functions on $X$ with the supremum norm $n(f)=\sup \{|f(x)|: x \in X\}$ and $\mu_{l}(u, v, t)=(1-t) \cdot u+t \cdot v$, for all $u, v \in B$ and $t \in \mathbb{I}$. Assume that for every two distinct points $a, b \in X$ there exists no more than one $\mu$-line that contains both of them. Consider the metrically embedding $\Phi: X \longrightarrow B$, defined by $\Phi(a)(x)=$ $d(x, a)-d(x, \omega)$ for all $a, x \in X$. Then $\Phi(\mu(a, b, t)) \neq \mu_{l}(\Phi(a), \Phi(b), t)$, for any two points $a, b \in X$ and $0<t<1$.

Proof. Fix two distinct points $a, b \in X$. Let $t \in \mathbb{I}$ and $0<t<1$. By virtue of Proposition 2.2 , there exists a unique $\mu$-line $(a b)_{\mu}$ that contains the points $a, b$.

Case 1. $0<t<1, c \notin(a b)_{\mu}$ and $d(a, c)=d(b, c)$.

We put $p=\mu(a, b, t)$. We have $d(p, c)>0$. We can assume that $a=\omega$. Then we put $\Phi(a)=f, \Phi(b)=g$ and $\Phi(p)=\psi$. By construction, $f(x)=0, g(x)=d(b, x)-d(a, x)$ and $\psi(x)=d(p, x)-d(a, x)$ for any $x \in X, g(b)=-d(a, b), g(c)=d(b, c)-d(a, c)=0, g(p)=$ $d(b, p)-d(a, p)=t \cdot d(a, b)-(1-t) \cdot d(a, b)=(2 t-1) \cdot d(a, b), \varphi(p)=-d(a, p)=-(1-t) \cdot d(a, b)$ $=(1-t) \cdot g(b)$ and, since $d(c, p)<d(c, a)=d(c, b), \varphi(c)=d(p, c)-d(a, c)=\lambda<0$. Hence $\Phi(\mu(a, b, t))=\Phi(p)=\psi \neq(1-t) \cdot g=\mu_{l}(f, g, t)=\mu_{l}(\Phi(a), \Phi(b), t)$.

Case 2. $0<t<1$ and $p=\mu(a, b, t)$.

Since $X$ is not a $\mu$-line, there exists a point $u \in X \backslash(a b)_{\mu}$. Obviously $(a b)_{\mu} \cap[a, u]_{\mu}=\{a\}$, $(a b)_{\mu} \cap[b, u]_{\mu}=\{b\}$ and $[a, u]_{\mu} \cap[b, u]_{\mu}=\{u\}$. We put $Y=[a, u]_{\mu} \cup[b, u]_{\mu}$. On $Y$ consider the continuous function $h(y)=d(a, y)-d(b, y)$. Since $h(a)=-d(a, b)<0, h(b)=d(a, b)>0$ and $Y$ is a connected space, there exists a point $p \in Y$ such that $h(p)=0$. By construction, $p \notin(a b)_{\mu}$ and $d(a, p)=d(b, p)$. By virtue of Case $1, \Phi(\mu(a, b, t)) \neq \mu_{l}(\Phi(a), \Phi(b), t)$ are not $\mu$-collinear and for $x, y \in X$ and $[u, v]_{\mu} \subseteq[x, y]_{\mu}$ it follows that $c \notin[x, y]_{\mu}$.

\section{IMMERSIONS AND EMBEDDINGS OF CONVEX METRIC SPACES}

Let $(X, d)$ be a metric space. A simple curve $\xi$ in $X$ is a continuous injection $\xi: \mathbb{I} \longrightarrow X$. We identify $\xi$ and the set $\xi(\mathbb{I}) \subseteq X$. A number $L(\xi)=\sup \Sigma\left\{d\left(\xi\left(t_{i}\right), \xi\left(t_{i+1}\right)\right): 0 \leq i \leq n\right\}$, where the supremum is taken over all sequences $0=t_{0}<t_{1}<\ldots, t_{n}<t_{n+1}=1$, is called the length of $\xi$. A simple curve $\xi$ is rectifiable, if the length $l(\xi)<+\infty$. 
A subspace $Y$ of the metric space $(X, d)$ is rectifiable, if for any two points $a, b \in Y$ there exists a rectifiable simple curve $\xi$ in $X$ such that $a, b \in \xi \subseteq Y$.

Let $Y$ be a rectifiable subspace of the metric space $(X, d)$. For any two points $a, b \in Y$ we put $d_{i n}(a, b)=\inf \{l(\xi): a, b \in \xi \subseteq Y, \xi$ is a simple rectifiable curve in $X\}$. Then $d_{i n}$ is the interior metric on $Y$ generated by the metric $d$.

Let $(Y, \rho)$ and $(X, d)$ be two metric spaces. A mapping $f: Y \longrightarrow X$ is called an isometrical immersion of the metric space $Y$ in the metric space $X$ if $f(Y)$ is a rectifiable subspace of the metric space $(X, d)$ and for the interior metric $d_{i n}$ on $f(Y)$ we have $\rho(y, z)$ $=d_{i n}(f(y), f(z))$, for all points $y, z \in Y$.

Remark 6.4. If $(F, \rho)$ is a metric space with a convex structure $\mu: F \times F \times \mathbb{I} \longrightarrow F$ and $g: F \longrightarrow B$ is an isometrically embedding of $F$ into a metric space $(B, d)$, then $g(F)$ is a rectifiable subspace of $B$ and $\rho(x, y)=d(g(x), g(y))=d_{i n}(g(x), g(y))$, for all $x, y \in F$, where $d_{i n}$ is the interior metric on $g(F)$. In particular, $g$ is an isometrical immersion.

Hilbert's theorem [18] states that there exists no complete regular surface $S$ of constant negative Gaussian curvature $K$ immersed in $\mathbb{R}^{3}$. This theorem answers the question for the negative case of which surfaces in $\mathbb{R}^{3}$ can be obtained by isometrically immersing complete manifolds with constant curvature. Distinct embedding problems were examined in $[1,8,14,15,16,24]$ and other important works. The Bolyai-Lobachevskii plane admits isometrically immersions in the Euclidean Space $\mathbb{R}^{4}$ (see [1]).

Theorem 6.2. Let $(B, n)$ be a strictly convex normed space of dimension $\geq 3$ and $\mu: L \times L \times \mathbb{I} \longrightarrow$ $L$ be the uniformly continuous hereditary strictly convex structure with convex round balls on the Bolyai-Lobacherskii plane $(L, \rho)$. Then any isometrically immersion $g: L \longrightarrow B$ is not an isometrically embedding of $(L, \rho)$ into the metric space $(B, d)$, where $d(x, y)=n(x-y)$, for all $x, y \in B$.

Proof. A normed vector space $(B, n)$ is strictly convex if and only it satisfies one of the following equivalent conditions:

1) if $x, y \in B, x \neq y$ and $n(x)=n(y)=1$, then $n(x+y)<2$;

2) if $x, y \in B, x \neq y$ and $n(x)=n(y)=1$, then $n(t \cdot x+(1-t) \cdot y)<1$, for all $0<t<1$.

Assume that $g: L \longrightarrow B$ is not an isometrically embedding of $(L, \rho)$ into the metric space $(B, d)$. We can identify a point $x \in L$ with the point $g(x) \in B$ and we consider that $L \subset B$ and $\rho(x, y)=d(x, y)=n(x-y)$ for all $x, y \in L$. For any two distinct points $x, y \in L$, denote by $(x y)_{L}$ the straight line passing through the points $x, y$ in the plane $L$ and $(x y)_{B}$ $=\{t \cdot x+(1-t) \cdot y: t \in \mathbb{R}\}$ is the vector or affine line passing through the points $x, y$ in the space $B$.

According to Proposition 3.3, $\mu_{L}: L \times L \times \mathbb{I} \longrightarrow L$ is the unique convex structure on the space $(L, \rho)$ which is hereditary strictly convex. Fix two distinct point $a, b \in L$ and $t \in \mathbb{I}$, where $0<t<1$. We put $\left.x_{t}=\mu_{(} a, b, t\right)$ and $y_{t}=(1-t) \cdot a+t \cdot b$. Then $d\left(a, y_{t}\right)+d\left(y_{t}, b\right)$ $=d(a, b), d\left(a, y_{t}\right)=t \cdot d(a, b), d\left(a, x_{t}\right)+d\left(x_{t}, b\right)=\rho\left(a, x_{t}\right)+\rho\left(x_{t}, b\right)=\rho(a, b)=d(a, b)$ and $d\left(a, x_{t}\right)=\rho\left(a, x_{t}\right)=t \cdot \rho(a, b)=t \cdot d(a, b)$. Since $B$ is strictly convex, we have $x_{t}=y_{t}$. Therefore $(a b)_{L}=(a b)_{B}$ for all distinct points $a, b \in L$. In this case $L$ is an affine (vector) plane in a linear space $B$ and any straight line of $L$ is an affine line of the linear space $B$, a contradiction, since we have distinct parallel postulates in the affine plane and in the Bolyai-Lobachevskii plane.

Corollary 6.1. The Bolyai-Lobacherskii plane and the Bolyai-Lobachevskii half-plane are not isometrically embedding in some strictly convex normed space.

Example 6.2. Let $(E, \rho)$ be a complete metric space and $\mu_{E}: E \times E \times \mathbb{I} \longrightarrow E$ a convex structure on the space $(E, \rho)$. Fix a normed space $(B, n)$ and an isometrically embedding 
of $(E, \rho)$ into $(B, n)$. We can assume that $E \subseteq B$ and $\rho(x, y)=n(x-y)$ for all points $x, y \in E$. Since $E$ is a complete metric space, $E$ is a closed subset of $B$. Fix two distinct points $a, b \in B$. We have four possible cases.

Case 1. $a, b \in E$.

We put $\mu(a, b, t)=\mu_{E}(a, b, t)$ for any $t \in \mathbb{I}$.

Case 2. $a, b \in B \backslash E$.

We put $\mu(a, b, t)=(1-t) \cdot a+t \cdot b$ for any $t \in \mathbb{I}$.

Case 3. $a \in E, b \in B \backslash E$ and $L \cap[a, d]=\{a\}$.

We put $\mu(a, b, t)=(1-t) \cdot a+t \cdot b$ and $\mu(b, a, t)=\mu(a, b, 1-t)$ for any $t \in \mathbb{I}$.

Case 4. $a \in E, b \in B \backslash E$ and $L \cap[a, d] \neq\{a\}$.

In this case there exists $c \in E$ such that $c \in E \cap[a, b]$ and $L \cap[c, b]=\{c\}$. let $p=$ $\rho(a, c) / \rho(a, b)$. Obviously, $c \notin\{a, b\}$ and $0<p<1$. We put $\mu(a, b, t)=\mu_{E}(a, b, t / p)$ for $t \leq p, \mu(a, b, t)=(1-t) \cdot a+t \cdot b$ for $t>p$ and $\mu(b, a, t)=\mu(a, b, 1-t)$.

The mapping $\mu: B \times B \times \mathbb{I} \longrightarrow B$ is a convex structure on $B$ which is an extension of the convex structure $\mu_{E}$.

Note that the extension $\mu$ of $\mu_{L}$ has some shortcomings. Firstly, it is not hereditary. Secondly, it is not always continuous.

Special isometrically embeddings of convex metric spaces are examined in [26].

Question. Are conditions (A), (B) and (C) from [36] sufficient for isometrically embedding the convex metric space in some Hilbert space?

Example 6.3. (Example 1 from [36]). Let $F$ be the family of closed intervals $[a, b]$ such that $0 \leq a \leq b \leq l$. For $A=[a, b], P=[p, q]$ from $F$ and $t \in \mathbb{I}$ we define a mapping $\mu_{F}$ by $\mu_{F}=$ $[(1-t) a+t p,(1-t) b=t q]$. Define a metric $d$ on $F$ by the Pompeiu-Hausdorff distance: $\rho(A, P)=\sup \{|\inf \{|c-u|: u \in A\}-\inf \{|c-v| ; v \in P\}|: c \in \mathbb{I}\}$.

We claim that $\rho(A, P)=\sup \{|a-p|,|b-q|\}$. To prove this, it is sufficient to consider three cases: $0 \leq a \leq b \leq p \leq q \leq 1 ; 0 \leq a \leq p \leq q \leq b \leq 1 ; 0 \leq a \leq p \leq b \leq q \leq 1$.

Denote by $B=\mathbb{R} \times \mathbb{R}=\{(x, y): x, y \in \mathbb{R}\}$ with the norm $n(x, y)=\sup \{|x|,|y|\}$. Then the mapping $\varphi: F \longrightarrow B$, where $\varphi([a, b])=(a, b)$ for any $[a, b] \in F$, is an isometrically embedding and $\varphi([a, b],[p, q], t)=(1-t) \cdot(a, b)+t \cdot(p, q)=((1-t) \cdot a+t \cdot p,(1-t) \cdot b+t \cdot q)$.

The following example is similar to the Example 2 from [35].

Example 6.4. Let $H$ be a Hilbert space of dimension $\geq 3$ (in particular an Euclidean space) with the inner product $\ll a, b \gg$. On $H$ consider the norm $n_{H}$ and the metric $d$ generated by the inner product: $\left(n_{H}(x)\right)^{2}=\ll x, x \gg$ and $d(x, y)=n_{H}(x-y)$, for all $x, y \in H$.

Fix the numbers $R>r>0$ and a vector $e \in H$ with $\ll e, e \gg=R^{2}$. We put $S=$ $\left\{x \in H: \ll x, x \gg=R^{2}\right\}, S_{0}=\left\{x \in S: \ll x-e, x-e \gg<2 \cdot R^{2}\right\}$ and $S_{r}=\{x \in S: \ll$ $\left.x-e, x-e \gg \leq 2 \cdot\left(R^{2}-R \cdot r\right)\right\}$. The set is a sphere of the radius $R$ with the centre at zero 0 , $S_{0}$ is an open half-sphere of $S$ and $S_{r}$ is a closed spherical cap of $S$. If $0<p<r<R$, then $S_{r} \subseteq S_{p} \subseteq S_{0} \subseteq S$. On $S$ and on the spherical caps of $S$ we consider the interior metric $d_{i n}$ generated by the metric $d_{H}$. Then $d_{i n}$ is the great-circle distance relatively to which the great-circles are the geodesic curves on $S$. For any two distinct points $a, b \in S_{0}$ there exists a unique geodesic curve $(a b)_{g}$ on $S$ through the points $a, b$ and we put $\mu_{S}(a, b, t)=$ $R \cdot((1-t) \cdot a+t \cdot b) / n_{H}((1-t) \cdot a+t \cdot b)$. Then $\mu_{S}$ is a convex structure on $S_{0}$ and $S_{r}$ relatively to metric $d_{i n}$. On $S_{r} \mu_{H}$ is a continuous hereditary strictly convex structure with convex round balls and $\gamma$-hyperconvex structure. From the Gauss-Bonnet theorem about geodesic triangle it follows that the spaces $\left(S_{0}, d_{i n}\right)$ and $\left(S_{r}, d_{i n}\right)$ are not isometrically embedding in some Hilbert space. 


\section{A SPECIAL CONSTRUCTION}

The following construction is inspired by the proof of Theorem 5 from [36].

Fix a compact space $S$ and a metric space $(E, d)$ with the continuous convex structure $\mu: E \times E \times \mathbb{I} \longrightarrow E$, i.e., the mapping $\mu: E \times E \times \mathbb{I} \longrightarrow E$ is continuous. Denote by $C(S, E)$ the space of all continuous mappings $f: S \longrightarrow E$ of the space $S$ into the space $E$. The mapping $f$ is bounded if the diameter $\operatorname{diam}_{d}(f(S))=\sup \{d(x, y): x, y \in f(S)\}<\infty$. Since $S$ is a compact space, any mapping $f \in C(S, E)$ is bounded. Consider the metric $\rho(f, g)=\sup \{d(f(x), g(x)): x \in S\}$ of uniform convergence. It is well known that the metric space $(C(S, E), \rho)$ is complete if and only if the metric space $(E, d)$ is complete. We identify the point $a \in E$ with the constant mapping $c_{a}: S \longrightarrow E$, where $c_{a}(x)=a$ for any $x \in S$. Then $E \subset C(S, E)$ and $d(a, b)=\rho(a, b)=\rho\left(c_{a}, c_{b}\right)$ for all $a, b \in E$.

On $C(S, E)$ we extend the convexity $\mu$ and we put $\eta(f, g, t)(x)=\mu(f(x), g(x), t)$, for all $f, g \in C(S, E), x \in S$ and $t \in \mathbb{I}$.

Property 1. $\eta(f, g, t) \in C(S, E)$ for all $f, g \in C^{0}(S, E)$ and $t \in \mathbb{I}$.

Proof. The set $F=\mu(f(S), g(S), \mathbb{I})$ is a compact subset of the metric space $E$. Then $\eta(f, g, t)(S) \subset F$ and $\eta(f, g, t)$ is a bounded function on $S$. The mapping $\varphi: E \times E \times \mathbb{I} \longrightarrow$ $X \times E \times \mathbb{I}$, where $\varphi(x, y, t)=(f(x), g(y), t)$ for any $(x, y, t) \in E \times E \times \mathbb{I}$, is continuous. The mapping $\varphi$ is uniformly continuous on $F \times F \times \mathbb{I}$. Hence $\eta(f, g, t) \in C(S, E)$ for each $t \in \mathbb{I}$. Property 2. Let $E$ be a compact space. Then $\eta: C(S, E) \times C)(S, E) \times \mathbb{I} \longrightarrow C(S, E)$ is a continuous convexity on the space $C(S, E)$.

Proof. Fix $f, g \in C(S, E), t \in \mathbb{I}$ and $\varepsilon>0$. Since the mapping $\mu$ is uniformly continuous, there exists a number $r>0$ such that $d(\mu(x, y, t), \mu(u, v, \tau)$ provided $(x, y, t),(u, v, \tau) \in$ $E \times E \times \mathbb{I}, d(x, u)<r, d(y, v)<r,|t-\tau|<r$. Let $0<\delta<r$. If $h, \psi \in C)(S, E), \tau \in \mathbb{I}$, then $d(\mu(f(x), g(x), t), \mu(h(x), \psi(x), \tau)<\varepsilon$ provided $\rho(f, h) \leq \delta, \rho(g, \psi) \leq \delta$ and $|t-\tau| \leq \delta$. Hence $\rho(\eta(f, g, t), \eta h, \psi, \tau) \leq \varepsilon$ provided $\rho(f, h) \leq \delta, \rho(g, \psi) \leq \delta$ and $|t-\tau| \leq \delta$.

We say that the convexity $\mu$ is non-expansive if $d(\mu(x, b, t), \mu(y, b, t)) \leq(1-t) d(x, y)$ for all $b, x, y \in E$ and $t \in \mathbb{I}$. In this case $d(\mu(a, x, t), \mu(a, y, t)) \leq t d(x, y)$ for all $a, x, y \in E$ and $t \in \mathbb{I}$.

The linear convexity of the normed linear space is non-expansive.

Property 3. If the convexity $\mu$ is non-expansive, then the convexity $\eta$ is non-expansive, too.

Proof. In this case $d(\eta(f, h, t), \eta(g, h, t))(x)=d(\mu(f(x), h(x), t), \mu(g(x), h(x), t)) \leq(1-t)$ $d(f(x), g(x))$ for all $f, g, h \in C(S, E), x \in S$ and $t \in \mathbb{I}$. Hence $d(\eta(f, h, t), \eta(g, h, t)) \leq$ $(1-t) d(f, g)$.

Let $k>0$ and $\left(S, d_{1}\right)$ be a metric space. Denote by $C^{k}(S, E)$ the family of all mappings $f: S \longrightarrow E$ with the property $d(f(x), f(y)) \leq k \cdot d_{1}(x, y)$, for all $x, y \in S$. Obviously, $C^{k}(S, E)$ is a closed subset of the space $C(S, E)$.

Property 4 . Let $\left(S, d_{1}\right)$ be a metric space, the convexity $\mu$ be non-expansive and $k>0$. Then $C^{k}(S, E)$ is a closed convex subset of the space $C(S, E)$. Moreover, if $E$ is a compact space, then $C^{k}(S, E)$ is a compact convex space, too.

Proof. If $f, g \in C^{k}(S, E)$ and $x, y \in S$, then $d(\eta(f, g, t)(x), \eta(f, g, t)(y))=d(\mu(f(x), g(x), t)$, $\mu(f(y), g(y), t)) \leq d(\mu(f(x), g(x), t), \mu(f(y), g(x), t))+d(\mu(f(y), g(x), t), \mu(f(y), g(y), t)) \leq$ $(1-t) d(f(x), f(y))+t d(g(x), g(y)) \leq(1-t) \cdot k \cdot d_{1}(x, y)+t \cdot k \cdot d_{1}(x, y)=k \cdot d_{1}(x, y)$. Therefore, $\eta(f, g, t) \in C^{k}(S, E)$ and $C^{k}(S, E)$ is a closed convex subset of the space $C(S, E)$.

Assume that $E$ is a compact convex space and let $r>0$ be given. A subset $L \subset S$ is called a $r$-net if $S=\cup\left\{B\left(x, r, d_{1}\right): x \in L\right\}$. For any metric space and for any $r>0$ there exists some $r$-net. A metric space is compact if it is complete and for each $r>0$ there exists a finite $r$-net.

Assume that $C^{k}(S, E)$ is not a compact metric space. Then there exists a number $\varepsilon>0$ such that the space $\left(C^{k}(S, E), \rho\right)$ has not a finite $\varepsilon$-net. Fix $r>0$ such that $0<4 \cdot(1+k) \cdot r<$ 
$\varepsilon$. Since $\left(S, d_{1}\right)$ is a compact metric space, there exists a finite $r$-net $L=\left\{s_{1}, s_{2}, \ldots, s_{m}\right\}$, where $m \in \mathbb{N}$. There exists an infinite sequence $\left\{g_{n} \in C^{k}(S, E): n \in \mathbb{N}\right\}$ such that $\rho\left(g_{n}, g_{q}\right) \geq \varepsilon$ for all distinct $n, q \in \mathbb{N}$.

Since $E$ is a compact space, there exist a finite subset $\left\{b_{1}, b_{2}, \ldots, b_{m}\right\}$ of the space $E$ and a finite family $\left\{\mathbb{N}_{i}: i \leq m\right\}$ of infinite subsets of the set $\mathbb{N}$ such that:

- $\mathbb{N}_{i+1}$ is a subset of the set $\mathbb{N}_{i}$ for each $i<m$;

- the sequence $\left\{g_{n}\left(s_{i}\right): n \in \mathbb{N}_{i}\right\}$ converges to a point $b_{i}$ for each $i \leq m$.

We can fix two distinct integers $n, q \in \mathbb{N}_{m}$ such that $d\left(b_{i}, g_{n}\left(s_{i}\right)\right)+d\left(b_{i}, g_{q}\left(s_{i}\right)<r\right.$ for each $i<m$. Hence $d\left(g_{n}\left(s_{i}\right), g_{q}\left(s_{i}\right)\right)<r$ for each $r \leq m$. Since $\rho\left(g_{n}, g_{q}\right) \geq \varepsilon$, there exists a point $s \in S$ such that $d\left(g_{n}(s), g_{q}(s)\right)>\varepsilon-r$. There exists $i \leq m$ such that $d_{1}\left(s, s_{i}\right)<r$. Then $d\left(g_{n}(s), g_{n}\left(s_{i}\right)\right) \leq k \cdot r, d\left(g_{q}(s), g_{q}\left(s_{i}\right)\right) \leq k \cdot r, d\left(g_{n}\left(s_{i}\right), g_{q}\left(s_{i}\right)\right)<r$ and $d\left(g_{n}(s), g_{q}(s)\right) \leq d\left(g_{n}(s), g_{n}\left(s_{i}\right)\right)+d\left(g_{n}\left(s_{i}\right), g_{q}\left(s_{i}\right)\right)+d\left(g_{q}\left(s_{i}\right), g_{q}(s)\right)<k \cdot r+r+k \cdot r<\varepsilon-r$, a contradiction. The proof is complete.

Property 5. If $\mu$ is a convex structure with convex round balls, then $\eta$ is a convex structure with convex round balls, too.

Proof. Fix $f, g, h \in C(S, E)$ and $t \in \mathbb{I}$. Then $\rho(h, \eta(f, g, t))=\sup \{d(h(x), \eta(f, g, t)(x))$ : $x \in S\}=\sup \{d(h(x), \mu(f(x), g(x), t)): x \in S\} \leq \sup \{d(h(x), f(x)), d(h(x), g(x)): x \in S\}$ $\leq \sup \{\rho(h, f), \rho(h, g)\}$. The proof is complete.

Property 6. Let $E$ be a compact space. If $\mu$ is a strictly convex structure with convex round balls, then $\eta$ is a strictly convex structure with convex round balls, too.

Proof. Fix $f, g, h \in C(S, E)$ and $t \in \mathbb{I} \backslash\{0,1\}$. The function $h(x, t)=\max \{\{\rho(h, f), \rho(h, g)\}-$ $d(h(x), \mu(f(x), g(x), t))$ is positive and continuous. Let $I_{n}=\left[2^{-n}, 1-2^{-n}\right]$. Since $E \times$ $I_{n}$ is a compact space then $h(x, t) \geq k_{n}>0$ for all $(x, t) \in E \times I_{n} . \rho(h, \eta(f, g, t))<$ $\sup \{\rho(h, f), \rho(h, g)\}$ for each $t \in \mathbb{I} \backslash\{0,1\}$.

For all $x y \in E$ and $t \in \mathbb{I}$ there exists a unique point $z \in E$ such that $d(x ; z)=(1-$ $t) d(x ; y)$ and $d(z ; y)=t d(x ; y)$. In this case $z=\mu(x, y, t)$. Fix $f, g \in C(S, E)$ and $t \in \mathbb{I}$. then $h(x)=\mu(f(x), g(x), t)$ is a unique continuous mapping of $S$ into $E$ such that $\rho(h, f)=$ $(1-t) \rho(f, g)$ and $\rho(h, g)=t \rho(f, g)$. The proof is complete.

Property 7. If $\mu$ is a T-convex structure, then $\eta$ is a T-convex structure, too.

Proof. Fix $f, g, h \in C(S, E)$ and $t \in \mathbb{I} \backslash\{0,1\}$. Then $\rho(h, \eta(f, g, t))=\sup \{d(h(x), \eta(f, g, t)(x))$ : $x \in S\}=\sup \{d(h(x), \mu(f(x), g(x), t)): x \in S\} \leq \sup \{t \cdot d(h(x), f(x))+(1-t) \cdot d(h(x)$, $g(x)): x \in S\} \leq\{t \cdot \rho(h, f)+(1-t) \cdot \rho(h, g)\}$. The proof is complete.

Property 8. Let $E$ be a compact space. If $\mu$ is a strongly T-convex structure, then $\eta$ is a strongly T-convex structure, too.

Proof. Fix $f, g, h \in C(S, E)$ and $t \in \mathbb{I}$. Then $\rho(h, \eta(f, g, t))=\sup \{d(h(x), \mu(f(x), g(x), t))$ : $x \in S\}<\sup \{t \cdot d(h(x), f(x))+(1-t) \cdot d(h(x), g(x)): x \in S\}$. Since $S$ is a compact space, $\rho(h, \eta(f, g, t))<\sup \{t \cdot d(h(x), f(x))+(1-t) \cdot d(h(x), g(x)): x \in S\} \leq\{t \cdot \rho(h, f)+$ $(1-t) \cdot \rho(h, g)\}$. The proof is complete.

Property 9. If $\mu$ is a uniformly continuous convex structure, then $\eta$ is a uniformly continuous convex structure, too.

Proof. Fix $\varepsilon>0$. Since $\mu$ is uniformly continuous, there exist $\delta, \xi \in \mathbb{R}$ such that $\varepsilon \geq \xi \geq$ $\delta>0$ and $d(\mu(x, y, t), \mu(s, z, t) \leq \xi$ provided $x, y, s, z \in E, t \in \mathbb{I}, d(x, s)<\xi$ and $d(y, z)<\delta$.

Fix $f, g, \varphi, \psi \in C(S, E), t \in \mathbb{I}$ for which $\rho(f, \varphi)<\xi$ and $\rho(g, \psi)<\delta$. Then $\rho(\eta(f, g, t), \eta(\varphi, \psi, t))$ $=\sup \{\mu(f(x), g(y), t), \varphi(x), \psi(x), t) \leq \xi$.

Property 10. $E$ is a convex closed subset of $C(S, E), d(a, b)=\rho(a, b)$ and $\mu(a, b, t)=\eta(a, b, t)$ for all $a, b \in E$ and $t \in \mathbb{I}$. If $s \in S$, then the mapping $R_{s}: C(S, E) \longrightarrow E$, where $R_{s}(f)=f(s)$ for any $f \in C(S, E)$, is a non-expansive retraction and $R_{s}(\eta(f, g, t))=\mu\left(R_{s}(f), R_{s}(g), t\right)$ for all $f, g \in C(S, E)$ and $t \in \mathbb{I}$.

Proof. By definition, the point $a \in E$ is identified with the constant function $c_{a}(s)=a$ for any $s \in S$. Then $R_{s}(a)=R_{s}\left(c_{a}\right)=a$ for any $a \in E$. Since $d(f(s), g(s)) \leq \rho(f, g)$, the 
mapping $R_{s}$ is non-expansive. By definition, $\eta(f, g, t)(s)=\mu(f(s), g(s), t)$. This completes the proof.

Remark 7.5. Supose that $(S, d)$ is a metric space with the continuous convex structure $\mu$. In [10] R. Cauty proved that for any continuous mapping $f: E \longrightarrow E$ the set $\{x \in E$ : $f(x)=x\}$ of fixed points of $f$ is non-empty.

That construction permits to extend Theorem 5 from [36].

Corollary 7.2. Suppose that $\left(S, d_{1}\right)$ is a metric space, $(E, d)$ is a compact space with a nonexpansive convexity $\mu$ and $k>0$. Fix a family $\mathcal{F}$ of non-expansive mappings of the space $C^{k}(S, E)$ into itself. If $\mathcal{F}$ is a left amenable semigroup of mappings or $\mathcal{F}$ is a family of mappings with invariant property in $C^{k}(S, E)$, then the family $\mathcal{F}$ has a common fixed point $f \in C^{k}(S, E)$.

Acknowledgments. The author wishes to express his hearty thanks to Professor Vasile Berinde for kind suggestions.

\section{REFERENCES}

[1] Aminov, Yu. A. Two-Dimensional Surfaces in 3-Dimensional and 4-Dimensional Euclidean Spaces. Results and Unsolved Problems. Ukr. Math. J. 71 (2019), no. 1, 1-38.

[2] Arhangel'skii, A. V. Mappings and spaces. Uspehi Mat. Nauk 21, no. 4 , 133-184; translated in English: Russ. Math. Surv. 21 (1966), no. 4, 115-162.

[3] Bakhtin, I. A. The contraction mapping principle in almost metric spaces. Functional analysis no. 30 (Russian), 26-37, Ulýanovsk. Gos. Ped. Inst., Ulýanovsk, 1989.

[4] Berinde, V. Error estimates for a class of $(\delta, \varpi)$-contractiones. Babeş - Bolyai Univ. Fac. Math. Comput. Sci. Res. Sem, Preprint no. 3, (1994), 29-38.

[5] Berinde, V.; Choban, M. Generalized metrics and their associate metrics. Relevance in metrical fixed point theory. Creat. Math. Inform. 22 (2013), no. 1, 11-18.

[6] Bridson, M. R.; Haefliger, A. Metric Spaces of Non-Positive Curvature. 319.Springer-Verlag, Berlin, 1999.

[7] Bula, I. Strictly convex metric spaces and fixed points. Mathematica Moravica 3 (1999), 5-16.

[8] Burago, D.; Ivanov, S. Isometric embeddings of Finsler manifolds. (Russian) Algebra i Analiz 5 (1993), no. 1, 179-192. translation in St. Petersburg Math. J. 5 (1994), no. 1, 159-169.

[9] Busemann, H. The geometry of geodesics Academic Press, 1955.

[10] Cauty, R. Un théorème de point fixe pour les fonctions multivoques acycliques. In: V. Kadets and W. Zelazko (editors), Functional Analysis and its Applications, Proceed. of the Intern. Conf. dedicated to 110th Anniversary of Stefan Banach, May 28-31, 2002, Lviv, Ukraine, Elsevier, 2004, 71-80.

[11] Chittenden, E. W. On the equivalence of Écart and voisinage. Trans. Amer. Math. Soc. 18 (1917), no. 2, 161-166.

[12] Choban, M. Selections and fixed points theorems for mapping defined on convex spaces. ROMAI J. 10 (2014), no. 2, 11-44.

[13] Choban, M. On the geometry of b-distances and the fixed points of mappings. Carpatian J. Math. 36 (2020), no. 2, 241-257.

[14] Demidov, S. S. (edit) Istoriko-matematicheskie issledovania (Historical and mathematical research). Vypusk (50), Moskva: Yanus-K, 2014.

[15] Efimov, N. V. Non-immersion of the Lobachevsky half-plane. Vestnik Moskov. Universiteta (1975), no. 2, 83-86.

[16] Heinonen, J. Geometric embeddings of metric spaces. Wikipedia, January 2003.

[17] Herstein, I. N.; Milnor, J. An axiomatic approach to measurable utility. Econometrica 21 (1953), $291-297$.

[18] Hilbert, D. Ueber flachen von constanter Gausscher krummung. Trans. Amer. Math. Soc. 2 (1901), 87-99.

[19] Fréchet, M. Sur quelques points du calcul fonctionnel. Rendiconti del Circolo Matematico di Palermo 22 (1906), $1-74$.

[20] Frink, A. H. Distance functions and the metrization problem. Bull. Amer. Math. Soc. 43 (1937), no. 2, $133-142$.

[21] Ješić, S. Fixed points theorem in spaces with general convex structure. Publ. Elektrotehn. Fak. Ser. Mat. 10 (1999), 107-112.

[22] Ješić, S. Fixed points and addmissible sets. Mathematica Moravica 7 (2003), 11-14.

[23] Joharinad, P.; Jost, J. Topology and curvature of metric spaces. Adv. Math. 356 (2019), 106813, 21 pp.

[24] Klotz-Milnor, T. Efimov's theorem about complete immersed surfaces of negative curvature. Usp. Mat. Nauk 41 (1986), no. 5, 3-57. 
[25] Kuratowski, C. Quelques problemes concernant les espaces metriques non-separables. Fundamenta Mathematicae 25 (1935), 534-545.

[26] Machado, H. V. A characterization of convex subsets of normed spaces. Kodai Math. Sem. Rep. 25 (1973), 307-320.

[27] Menger, K. Untersuchungen über allgemeine Metrik. Math. Ann. 100 (1928), 75-163.

[28] Michael, E. Convex structures and continuous selections. Canad. J. Math. 11 (1959), 556-575.

[29] Nedev, S. I. o-metrizable spaces. Trudy Moskov. Mat. Obsc. 24 (1971), 201-236; English translation: Trans. Moscow Math. Soc. 24 (1974), 213-247.

[30] Nedev, S.; Choban, M., On the theory of o-metrizable spaces. I. (Russian) Vestnik Moskov. Univ. Ser. I Mat. Meh. 27 (1972), no. 1, 8-15.

[31] Nedev, S.; Choban, M. On the theory of o-metrizable spaces. II. (Russian) Vestnik Moskov. Univ. Ser. I Mat. Meh. 27 (1972), no. 2, 10-17.

[32] Nedev, S. and Choban, M., On the theory of o-metrizable spaces. III (Russian) Vestnik Moskov. Univ. Ser. I Mat. Meh. 27 (1972), no. 3, 10-15.

[33] Niemytzki V. On the third axiom of metric spaces.Trans Amer. Math. Soc. 29 (1927), 507-513.

[34] Nijenhuis, A. A note on hyperconvexity in Riemannian manifolds. Canad. J. Math. 11 (1959), 576-582.

[35] Shimizu, T.; Takahashi, W. Fixed points of multivalued mappings in certain metric spaces. Topol. Methods Nonlinear Anal. 8 (1996), no. 1, 197-203.

[36] Takahashi, W. A convexity in metric space and nonexpansive mappings I. Kodai Math. Sem. Rep. 22 (1970), 142-149.

[37] Tasković, M. General convex topological spaces and fixed points. Math. Moravica 1 (1997), 127-134.

[38] Kuwae, K.; Shioya, T. On generalized measure contraction property and energy functionals over Lipschitz maps. Potential Analysis 15 (2001), 105-121.

[39] Skorneakov, L. A. Stochastic algebra. Izvestia Vyschih Ucheb. Zavedenii, Matematica 7 (1985), 3-11; English translation: Soviet Mathematics (Izvestiya VUZ, Matematika) 29 (1985), no. 7, 1-12.

[40] Vel, M. van de Theory of Convex Structures. North-Holland, Amsterdam, 1993.

[41] Wilson, W. A. On semi-metric spaces. Amer. J. Math. 53 (1931), no. 2, 361-373.

Department of Algebra, Geometry and Topology

TiRAspol StATE UNIVERSITY, CHIŞINĂU, REPUbliC OF MOLdOVA

Email address: mmchoban@gmail. com 